\title{
Tetrandrine ameliorates sevoflurane-induced cognitive impairment via the suppression of inflammation and apoptosis in aged rats
}

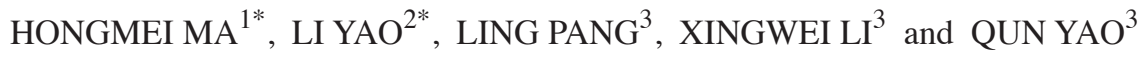 \\ Departments of ${ }^{1}$ Training, ${ }^{2}$ Genecology and ${ }^{3}$ Anesthesiology, Zaozhuang Municipal Hospital, \\ Zaozhuang, Shandong 277100, P.R. China
}

Received March 8, 2015; Accepted December 1, 2015

DOI: $10.3892 / \mathrm{mmr} .2016 .5132$

\begin{abstract}
Tetrandrine is a bisbenzylisoquinoline alkaloid extracted from Stephania tetrandra, a traditional Chinese herbal medicine, which has been observed to exert anti-inflammatory effects. The aim of the current study was to investigate whether tetrandrine was able to ameliorate sevoflurane-induced cognitive impairment in aged rats. Male 20-month-old Sprague-Dawley rats underwent sevoflurane-induction in an environment containing $2 \%$ sevoflurane for $5 \mathrm{~h}$. The Morris water maze test was used to measure the effect of tetrandrine on learning and memory in sevoflurane-treated aged rats. Western blot analysis of the protein expression levels of cyclooxygenase-2 (COX-2), inducible nitric oxide synthase (iNOS) and Bcl-2 was conducted. ELISAs were used to measure the levels of interleukin (IL)-1 $\beta$, tumor necrosis factor (TNF- $\alpha$ ), nuclear factor- $\kappa \mathrm{B}(\mathrm{NF}-\kappa \mathrm{B})$ and caspase-3. In the present study, tetrandrine improved the learning and memory deficits observed in sevoflurane-treated aged rats. Treatment with tetrandrine reduced the expression levels of COX-2, IL- $1 \beta$, TNF- $\alpha$, NF- $\kappa B$, iNOS and caspase-3, and increased the $\mathrm{Bcl}-2$ protein expression in sevoflurane-treated aged rats. In conclusion, the current study indicated that tetrandrine ameliorates sevoflurane-induced cognitive impairment via the suppression of inflammation and apoptosis in aged rats. Thus, tetrandrine may be a potential novel candidate to protect against the effects of sevoflurane anesthesia on cognitive function.
\end{abstract}

Correspondence to: Ms. Qun Yao, Department of Anesthesiology, Zaozhuang Municipal Hospital, 41 Longtou Road, Zaozhuang, Shandong 277100, P.R. China

E-mail: yaoqun77777@163.com

*Contributed equally

Key words: tetrandrine, sevoflurane, cognitive impairment, apoptosis, inflammation

\section{Introduction}

Central nervous system complications occurring following general anesthesia are receiving increased research focus. Post-operative cognitive dysfunction (POCD) occurs within a few days of anesthesia and affects cognitive ability, memory and sleep in a reversible manner (1). Sevoflurane is an inhaled anesthetic with rapid induction, and minimal effects on the respiratory tract, circulation and metabolic rate $(2,3)$. In elderly patients, with brain oxygen metabolism imbalance related to postoperative cognitive dysfunction; Sevoflurane anesthesia in one-lung ventilation can cause cerebral oxygen metabolism abnormalities, one-lung ventilation after anesthesia in elderly patients with cognitive function is ignored (4). In the pathogenesis of POCD, inflammatory reactions and neuronal apoptosis are suggested to serve important roles (5). Previous studies have indicated that inflammatory reactions in POCD occur as a result of apoptotic injury $(6,7)$.

Tetrandrine is a bisbenzylisoquinoline alkaloid extracted from Stephania tetrandra, a traditional Chinese herbal medicine, and has been used for diseases including hypertension, arrhythmia and pulmonary fibrosis (8). In recent years, a study indicated that tetrandrine is able to inhibit the growth of a variety of tumor cells, and as a result research has focused on its potential use as an anticancer drug (9). However, it remains to be elucidated whether tetrandrine is able to affect sevoflurane-induced cognitive impairment in aged rats. In the current study, the effect of tetrandrine on sevoflurane-induced cognitive impairment was investigated in aged rats and the potential mechanisms explored.

\section{Materials and methods}

Animals and surgery. Male 20-month-old Sprague-Dawley rats were obtained from Shandong Hongli Medical Animal Experiment Research Co., Ltd. (Shandong, China) and used in the present study, and were acclimated in the animal care facility for 1 week prior to the study. All rats were maintained in a 12:12 $\mathrm{h}$ light-dark cycle with a constant room temperature $\left(23 \pm 1^{\circ} \mathrm{C}\right)$ and allowed continuous access to food and water. The experimental protocol was approved by the Animal Ethics Committee of Zaozhuang Municipal Hospital (Shandong, 
China). Rats were anesthetized in an environment containing $2 \%$ sevoflurane (Sigma-Aldrich, St. Louis, MO, USA) for $5 \mathrm{~h}$. The total gas flow was $1.5 \mathrm{l} / \mathrm{min}$, using $70 \% \mathrm{O}_{2}$ as a carrier. The oxygen and anesthetic agent fractions were measured using a gas analysis system (TH-890B; Wuhan Tianhong Instruments Co., Ltd., Wuhan, China).

Experimental groups. A total of 32 rats were randomly divided into four groups with 8 animals in each group: i) Control group (Con), normal rats injected with saline $(0.1 \mathrm{ml} / 100 \mathrm{~g}$ intraperitoneally, i.p.); ii) Control-tetrandrine group (C-Tet), normal rats received tetrandrine $(30 \mathrm{mg} / \mathrm{kg}$ body weight, i.p., every 3 days; Sigma-Aldrich) for 4 consecutive weeks; iii) Sevoflurane group (Seo), sevoflurane-induced rats were anesthetized in an environment containing $2 \%$ sevoflurane for $5 \mathrm{~h}$, and received saline $(0.1 \mathrm{ml} / 100 \mathrm{~g}$, i.p.); iv) Sevoflurane-tetrandrine group (S-Tet), sevoflurane-induced rats were anesthetized in an environment containing $2 \%$ sevoflurane for $5 \mathrm{~h}$, and received tetrandrine $(30 \mathrm{mg} / \mathrm{kg}$ body weight, i.p., every 3 days) for 4 consecutive weeks. The structure of tetrandrine (purity, $>99 \%$ ) is presented in Fig. 1.

Morris water maze (MWM). Following treatment with tetrandrine, the spatial memory ability of rats was investigated using the MWM test. A circular, black painted pool $(180 \mathrm{~cm}$ diameter, $50 \mathrm{~cm}$ deep) was filled with water containing black non-toxic ink (temperature at $25 \pm 1^{\circ} \mathrm{C}$ ) to a depth of $30 \mathrm{~cm}$. A hidden platform (10 cm diameter) was submerged $1 \mathrm{~cm}$ below the water line and placed in the center of the northeast quadrant, which was determined with four starting locations called north $(\mathrm{N})$, east $(\mathrm{E})$, south $(\mathrm{S})$ and west $(\mathrm{W})$ at equal distance around the rim. The rats were tested 4 times/day over 4 consecutive days. The time taken to escape to the hidden platform was recorded as escape latency. Mean path length was measured as the mean distance of movement for each rat. Time spent in the target quadrant was recorded using a stopwatch. In turn, each rat was placed in a random starting position, facing the pool wall and allowed to swim and self-discover the hidden platform. Once the rat arrived at the platform, it was allowed to stay on it for $30 \mathrm{sec}$. On the 5th day, the hidden platform was removed, and each rat was allowed to swim freely for $60 \mathrm{sec}$. The number of times the rat crossed the platform was recorded within $60 \mathrm{sec}$ and swimming speed was calculated as the distance $\mathrm{x}$ number of times crossing platform (n)/60 sec.

Western blot analysis. Following treatment with tetrandrine, the rats were anesthetized with $2 \%$ sevoflurane (Sigma-Aldrich) and were sacrificed by spinal dislocation, and the brain tissue was obtained. The brain tissue was homogenized in radioimmunoprecipitation assay lysis buffer (Sangon Biotech Co., Ltd., Shanghai, China) containing $1 \mathrm{mM}$ ethylenediaminetetraacetic acid, $50 \mathrm{mM}$ Tris- $\mathrm{HCl}, 150 \mathrm{mM}$ $\mathrm{NaCl}, 0.1 \%$ sodium dodecyl sulfate (SDS; Nanjing Sunshine Biotechnology Co., Ltd., Nanjing, China), 0.25\% sodium deoxycholate (Nanjing Sunshine Biotechnology Co., Ltd.), 1\% NP-40 (Nanjing Sunshine Biotechnology Co., Ltd.), a protease inhibitor cocktail and phosphatase inhibitors (both Sangon Biotech Co., Ltd.). Following homogenization

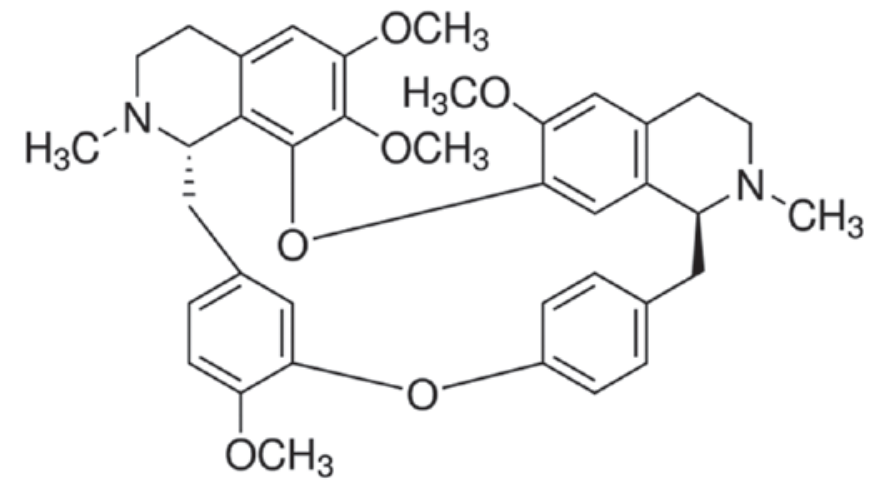

Figure 1. Chemical structure of tetrandrine.

$\left(12,000 \mathrm{x} \mathrm{g}, 4^{\circ} \mathrm{C}, 10 \mathrm{~min}\right)$, the clear upper supernatants were collected, and the total protein measured using a bicinchoninic acid (BCA) protein assay (Beyotime Institute of Biotechnology, Shanghai, China). Equal amounts of proteins were loaded onto a $10 \%$ SDS-PAGE gel (Nanjing Jiancheng Bioengineering Institute, Nanjing, China) for electrophoresis and then transferred onto nitrocellulose membranes (EMD Millipore, Billerica, MA, USA). Membranes were blocked with $5 \%$ (w/v) skimmed milk in $0.1 \%$ (v/v) Tween 20 (Nanjing Jiancheng Bioengineering Institute) in phosphate-buffered saline (PBST) for $2 \mathrm{~h}$. The membranes were then incubated with rabbit polyclonal anti-cyclooxygenase-2 (COX-2; 1:1,000; cat. no. D151772; Sangon Biotech Co., Ltd.), mouse monoclonal anti-inducible nitric oxide synthase (iNOS; 1:1,000; cat. no. sc-5302; Santa Cruz Biotechnology, Inc., Dallas, TX, USA), mouse monoclonal anti-Bcl-2 (1:500; cat. no. sc-509; Santa Cruz Biotechnology, Inc.) and rabbit polyclonal anti- $\beta$-actin (1:5,000; cat. no. D110007; Sangon Biotech Co., Ltd.) antibodies overnight at $4^{\circ} \mathrm{C}$ with agitation. Antibody recognition was detected using anti-rabbit and anti-mouse antibody (1:500 and 1:5,000 in PBS containing $1 \%$ normal goat serum, respectively; cat. nos. sc-2491 and sc-358922, respectively; Santa Cruz Biotechnology, Inc.). Antibody-bound proteins were detected using an enhanced chemiluminescence western blotting analysis system (Vector Laboratories, Inc., Burlingame, CA, USA).

Enzyme-linked immunosorbent assays (ELISA). Following treatment with tetrandrine, the brain tissue was homogenized in radioimmunoprecipitation assay lysis buffer. The homogenate was centrifuged at $12,000 \mathrm{x}$ g for $15 \mathrm{~min}$ at $4^{\circ} \mathrm{C}$. The clear upper supernatants were collected for analysis of interleukin (IL)-1 $\beta$, tumor necrosis factor- $\alpha$ (TNF- $\alpha$ ) and nuclear factor $-\kappa \mathrm{B}(\mathrm{NF}-\kappa \mathrm{B})$ activities according to the manufacturer's instructions (Nanjing KeyGen Biotech Co., Ltd., Nanjing, China).

Measurement of caspase-3 activity. Following treatment with tetrandrine, the brain tissue was homogenized in radioimmunoprecipitation assay lysis buffer. The homogenate was centrifuged at $12,000 \mathrm{x}$ g for $15 \mathrm{~min}$ at $4^{\circ} \mathrm{C}$, and the clear upper supernatants collected and the total protein content was measured using a BCA protein assay (Beyotime Institute of Biotechnology). Equal amounts of protein were added to the reaction buffer containing 
Ac-DEVD-pNA ( $2 \mathrm{mM}$; Beyotime Institute of Biotechnology) and incubated at $37^{\circ} \mathrm{C}$ for $6 \mathrm{~h}$. Caspase-3 activation was measured using a Microplate reader (EMR500; Labomed, Inc., Los Angeles, CA, USA) at an absorbance of $405 \mathrm{~nm}$.

Statistical analysis. Data are presented as the mean \pm standard deviation of three independent experiments. Differences between groups were investigated using SPSS software, version 19.0 (IBM SPSS, Armonk, NY, USA) and a one-way analysis of variance followed by Dunnett's test. $\mathrm{P}<0.05$ was considered to indicate a statistically significant difference.

\section{Results}

Tetrandrine improves learning and memory deficits in sevoflurane-treated aged rats. In order to investigate the effects of tetrandrine on learning and memory in sevoflurane-treated aged rats, learning and memory were measured using MWM tests. Fig. 2A shows that the escape latency was increased following treatment with sevoflurane, compared with the control group. However, treatment with tetrandrine reduced the escape latency in sevoflurane-treated aged rats (Fig. 2A). In addition, in the sevoflurane group, the mean path length was increased compared with the control group (Fig. 2B). Pretreatment with tetrandrine significantly reduced the mean path length in sevoflurane-treated aged rats $(\mathrm{P}<0.01$; Fig. $2 \mathrm{~B})$.

As presented in Fig. $2 \mathrm{C}$ and D, sevoflurane significantly reduced the time the rats spent in the target quadrant and the number of times the rats crossed the platform location, compared with the control group. Notably, treatment with tetrandrine improved these alterations in sevoflurane-treated rats (Fig. 2C and D). However, no significant alterations in swimming speed were observed in any of the groups (Fig. 2E). Therefore, tetrandrine at $30 \mathrm{mg} / \mathrm{kg}$ was observed to provide protection against sevoflurane-induced cognitive impairment in rats.

Tetrandrine reduces COX-2 expression in sevoflurane-treated aged rats. In order to investigate the protective effect of tetrandine in sevoflurane-treated aged rats, the expression levels of COX-2 were measured using western blot analysis. Western blotting indicated that COX-2 protein expression was upregulated following sevoflurane treatment (Fig. 3A and B). Co-treatment of rats with tetrandrine significantly reduced $\mathrm{COX}-2$ protein expression $(\mathrm{P}<0.01$; Fig. $3 \mathrm{~A}$ and $\mathrm{B})$.

Tetrandrine reduces the levels of $I L-1 \beta$ and TNF- $\alpha$ in sevoflurane-treated aged rats. To investigate the protective effects of tetrandrine on inflammation in sevoflurane-treated aged rats, the expression levels of IL- $1 \beta$ and TNF- $\alpha$ were measured. This indicated an increase in IL- $1 \beta$ and TNF- $\alpha$ production in the sevoflurane-treated aged rats compared with the control group (Fig. 4A and B). Compared with the sevoflurane group rats, the levels of IL- $1 \beta$ and TNF- $\alpha$ were significantly reduced in the sevoflurane-tetrandrine group $(\mathrm{P}<0.01$; Fig. $4 \mathrm{~A}$ and B).

Tetrandrine reduces $N F-\kappa B$ in sevoflurane-treated aged rats. To further investigate the protective effects of tetrandrine on
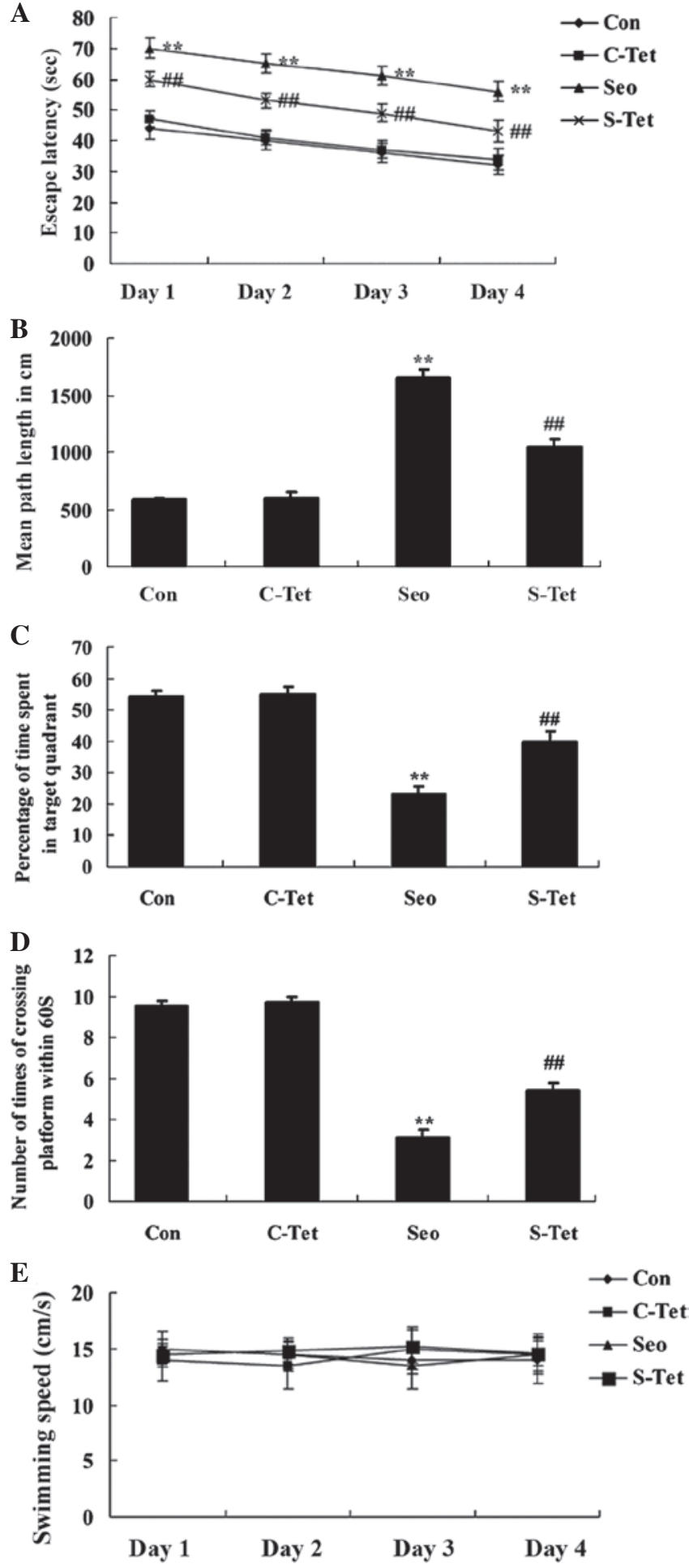

Figure 2. Tetrandrine ameliorates learning and memory deficits in sevoflurane-induced aged rats. Effect of tetrandrine on (A) the escape latency, (B) mean path length, $(C)$ mean percentage of time spent in the target quadrant, (D) the number of times of crossing the platform and (E) swimming speed in sevoflurane-treated aged rats. Values are presented as the mean \pm standard deviation. ${ }^{* *} \mathrm{P}<0.01$ vs. the Con group; ${ }^{\# \#} \mathrm{P}<0.01$ vs. the Seo group. Con, Control group; C-Tet, Control-tetrandrine group; Seo, Sevoflurane group; S-Tet, Sevoflurane-tetrandrine group.

inflammation in sevoflurane-treated aged rats, the activity of $\mathrm{NF}-\mathrm{\kappa B}$ was measured. As presented in Fig. 5, the activity of NF- $\kappa \mathrm{B}$ was increased by sevoflurane compared with the control. However, tetrandrine significantly reduced the 


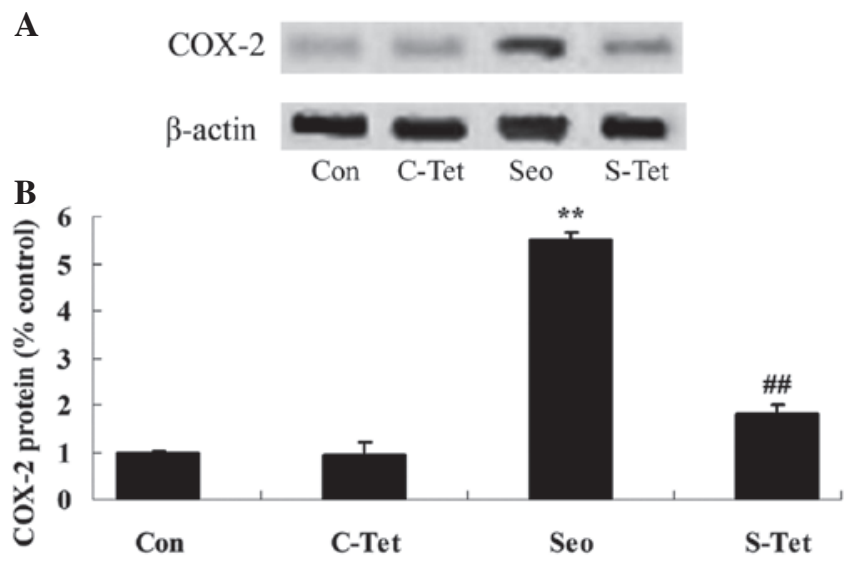

Figure 3. Tetrandrine reduces COX-2 expression level in sevoflurane-treated aged rats. (A) Western blot images and (B) quantification indicating that tetrandrine reduces COX-2 protein expression. Values are presented as the mean \pm standard deviation. ${ }^{* *} \mathrm{P}<0.01$ vs. the Con group; ${ }^{\# \#} \mathrm{P}<0.01$ vs. the Seo group. COX-2, cyclooxygenase 2; Con, Control group; Seo, Sevoflurane group; C-Tet, Control-tetrandrine group; S-Tet, Sevoflurane-tetrandrine group.

activity of NF- $\mathrm{NB}$ in sevoflurane-treated aged rats $(\mathrm{P}<0.01$; Fig. 5).

Tetrandrine reduces iNOS expression levels in sevoflurane-treated aged rats. To further investigate the protective effects of tetrandrine on sevoflurane-treated aged rats, iNOS protein expression was measured by western blot analysis. The protein expression of iNOS was increased in the sevoflurane group compared with the control group (Fig. 6A and B). Following tetrandrine treatment, iNOS protein expression was significantly reduced in the sevoflurane-treated aged rats $(\mathrm{P}<0.01$; Fig. $6 \mathrm{~A}$ and $\mathrm{B})$.

Tetrandrine reduces caspase-3 activity in sevoflurane-treated aged rats. To investigate whether alterations in apoptosis may serve a role in the protective effects of tetrandrine on sevoflurane-treated aged rats, the activity of caspase- 3 was measured. As presented in Fig. 7, sevoflurane significantly increased the activity of caspase-3 compared with the control group. However, treatment with tetrandrine significantly reduced the increase in caspase-3 activity in sevoflurane-treated aged rats $(\mathrm{P}<0.01$; Fig. 7$)$.

Tetrandrine reduces Bcl-2 expression levels in sevoflurane-treated aged rats. To further investigate the protective effects of tetrandrine on apoptosis in sevoflurane-treated aged rats, Bcl-2 protein expression was measured using western blot analysis. As presented in Fig. 8A and B, sevoflurane reduced the expression levels of $\mathrm{Bcl}-2$, compared with the control group. However, tetrandrine resulted in an increase in the expression levels of Bcl-2 in sevoflurane-treated aged rats $(\mathrm{P}<0.01$; Fig. $8 \mathrm{~A}$ and $\mathrm{B})$.

\section{Discussion}

POCD is a common complication in elderly patients following surgery, and presents clinically as cognitive dysfunction, memory impairment, personality alterations and problems with
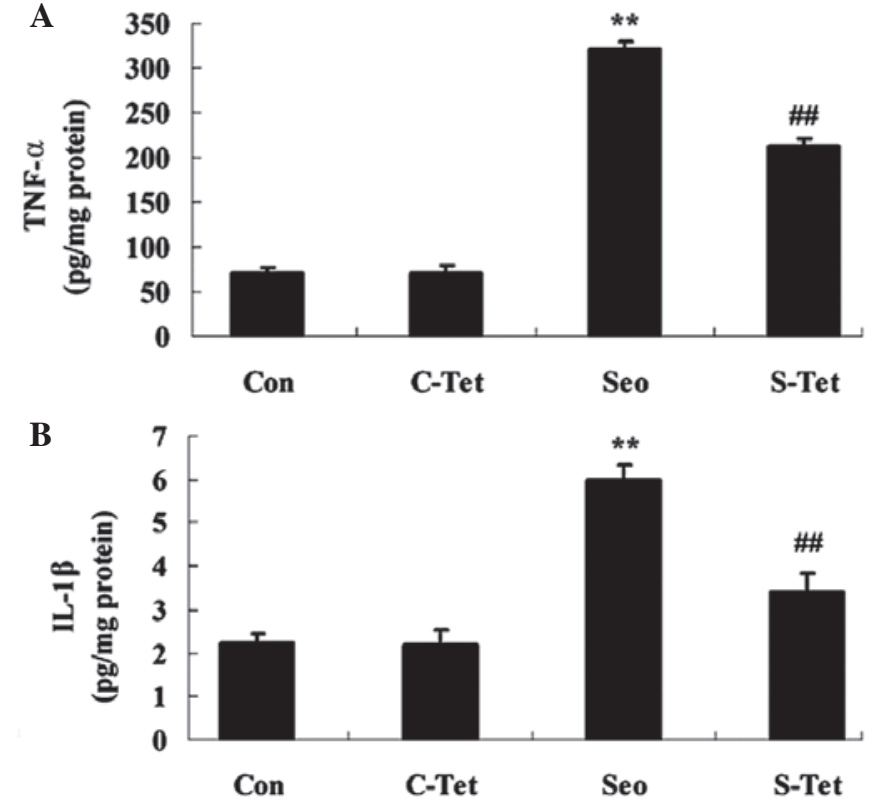

Figure 4. Tetrandrine reduces the levels of (A) IL-1 $\beta$ and (B) TNF- $\alpha$ in sevoflurane-treated aged rats. Values are presented as the mean \pm standard deviation. ${ }^{* *} \mathrm{P}<0.01$ vs. the Con group; ${ }^{\# \#} \mathrm{P}<0.01$ vs. the Seo group. IL-1 $\beta$, interleukin-1 $\beta$; TNF- $\alpha$; tumor necrosis factor- $\alpha$; Con, Control group; Seo, Sevoflurane group; C-Tet, Control-tetrandrine group; S-Tet, Sevoflurane-tetrandrine group.

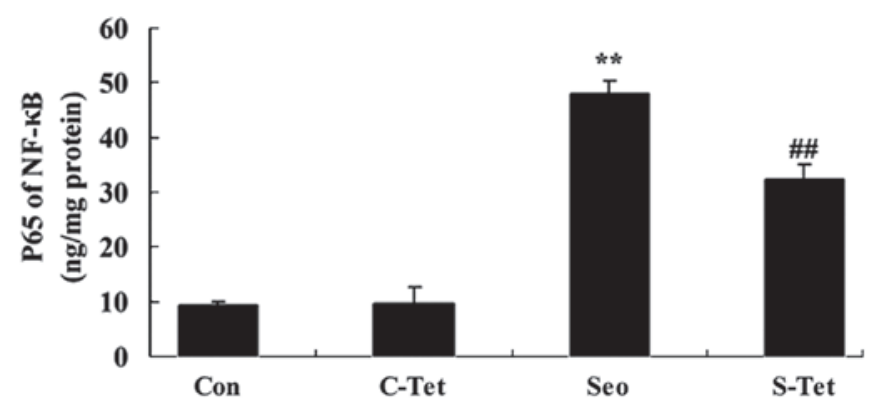

Figure 5. Tetrandrine reduces $\mathrm{NF}-\kappa \mathrm{B}$ in sevoflurane-treated aged rats. Values are presented as the mean \pm standard deviation. ${ }^{* *} \mathrm{P}<0.01$ vs. the Con group; ${ }^{\#} \mathrm{P}<0.01$ vs. the Seo group. NF- $\kappa \mathrm{B}$, nuclear factor- $\kappa \mathrm{B}$; Con, Control group; Seo, Sevoflurane group; C-Tet, Control-tetrandrine group; S-Tet, Sevoflurane-tetrandrine group.

social integration (10). POCD is associated with anesthesia. Anesthesia results in central nervous system dysfunction, via alterations in acetylcholine, dopamine and catecholamines (11). Sevoflurane is an inhaled anesthetic which results in quick induction of anesthesia and has been widely used clinically. However, studies have indicated that sevoflurane anesthesia may lead to POCD in elderly patients $(12,13)$. Tetrandrine is a bisbenzylisoquinoline alkaloid extracted from Stephania tetrandra, a traditional Chinese herbal medicine, and has been demonstrated to posses anti-inflammatory, analgesic and antihypertensive effects, and to block calcium channels (14-17). In the current study, tetrandrine was demonstrated to improve the learning and memory deficits observed in sevoflurane-treated aged rats. Chen et al (18) reported that tetrandrine was able to ameliorate cognitive impairment in a rat model of chronic cerebral hypoperfusion. In addition, He et al (19) demonstrated that tetrandrine attenuated 
A

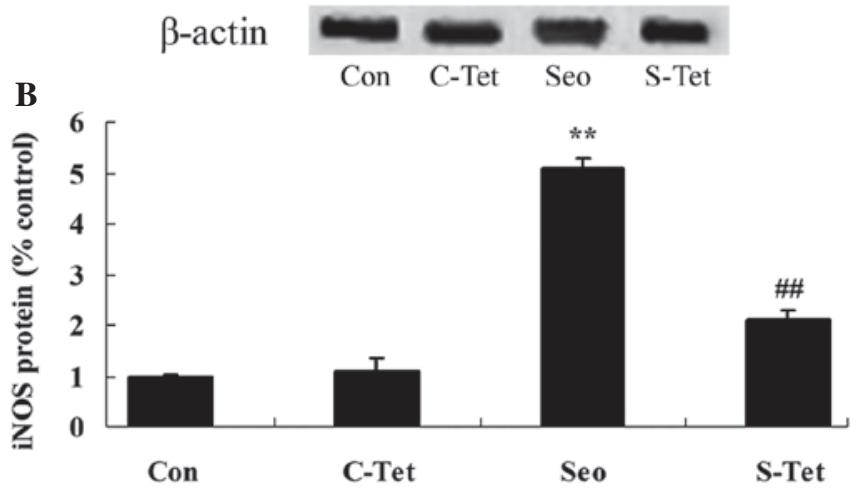

Figure 6. Tetrandrine reduces iNOS in sevoflurane-induced aged rats (A) Western blotting images and (B) quantification of iNOS protein expression levels in sevoflurane-induced aged rats. Values are presented as the mean \pm standard deviation. ${ }^{* *} \mathrm{P}<0.01$ vs. the Con group; ${ }^{\# \#} \mathrm{P}<0.01$ vs. the Seo group. iNOS, inducible nitric oxide synthase; Con, Control group; Seo, Sevoflurane group; C-Tet, Control-tetrandrine group; S-Tet, Sevoflurane-tetrandrine group.

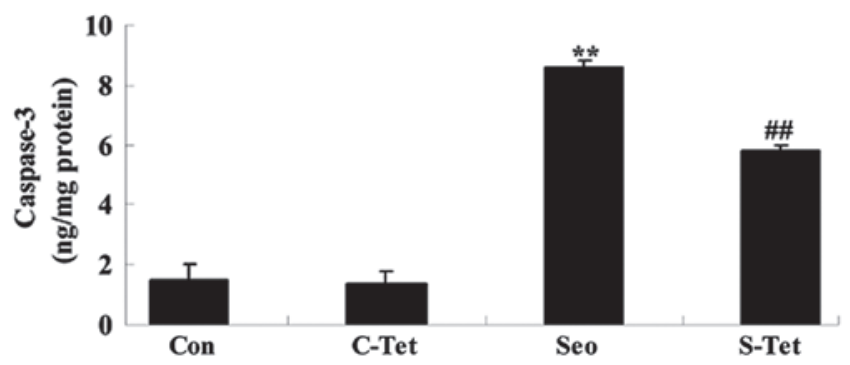

Figure 7. Tetrandrine reduces caspase- 3 in sevoflurane-induced aged rats. Values are presented as the mean \pm standard deviation. ${ }^{* *} \mathrm{P}<0.01$ vs. the Con group; ${ }^{\# \#} \mathrm{P}<0.01$ vs. the Seo group. Con, Control group; Seo, Sevoflurane group; C-Tet, Control-tetrandrine group; S-Tet, Sevoflurane-tetrandrine group.

spatial memory impairments in a rat model of Alzheimer's disease (19). Thus, tetrandrine may represent a potential candidate for the treatment of sevoflurane-induced cognitive impairment.

Inflammatory reactions, the activation of $\mathrm{COX}-2$, and increased prostaglandin levels are closely associated processes in dentate gyrus granular cells, hippocampal pyramidal neurons, the pear-shaped zone, the new shallow cortex cell layer, the lower part of the striatum, and the thalamus of POCD rats (20-22). A previous study indicated that COX-2-dependent mechanisms may serve a role in POCD (20). In the present study, tetrandrine reduced COX-2 protein expression in sevoflurane-treated aged rats. In support of this, Wu et al (23) reported that tetrandrine treatment reduced COX-2 expression in human monocytic cells. Kang et al (24) indicated that tetrandrine suppressed the production of pro-inflammatory mediators via the suppression of COX-2 expression in stimulated mast cells (24). However, the effect of various doses of tetrandrine on sevoflurane-induced COX-2 expression requires further investigation.

POCD is a common complication in elderly patients following surgery. The mechanism of POCD is not fully
A

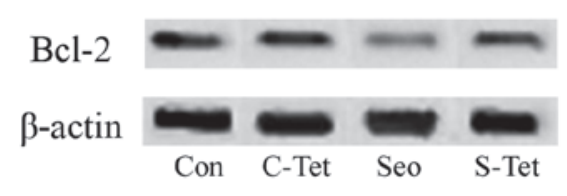

B

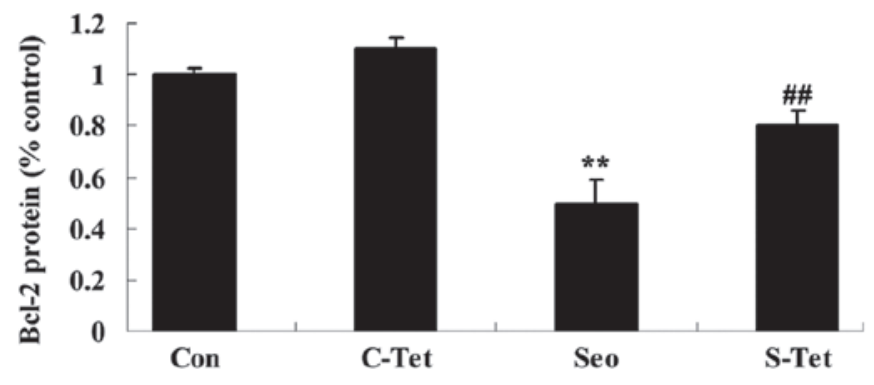

Figure 8. Tetrandrine reduces the expression levels of Bcl-2 in sevoflurane-treated aged rats. (A) Western blotting images and (B) quantification of $\mathrm{Bcl}-2$ protein expression levels in sevoflurane-treated aged rats. Values are presented as the mean \pm standard deviation. ${ }^{* *} \mathrm{P}<0.01$ vs. the Con group; ${ }^{\# \#} \mathrm{P}<0.01$ vs. the Seo group. Con, Control group; Seo, Sevoflurane group; C-Tet, Control-tetrandrine group; S-Tet, Sevoflurane-tetrandrine group.

understood, however a previous study suggests that inflammation within the central nervous system may serve an important role (25). Peripheral inflammation is able to result in inflammatory responses in the central nervous system via a variety of mechanisms, leading to neuronal dysfunction, inhibition of neuronal regeneration and the induction of apoptosis, ultimately resulting in reduced cognitive function (26). In addition, the present study demonstrated that tetrandrine is able to reduce the levels of IL-1 $\beta$, TNF- $\alpha$ and NF- $\kappa \mathrm{B}$ in sevoflurane-treated aged rats. A study by Lin et al (27) indicated that tetrandrine suppressed LPS-induced astrocyte activation via inhibition of IL- $1 \beta$, TNF- $\alpha$ and NF- $\kappa$ B signaling. In addition, Zhang et al (28) reported that administration of tetrandrine significantly reduced IL- $1 \beta, \mathrm{TNF}-\alpha$ and NF- $\kappa$ B levels in a mouse model of ulcerative colitis.

Nitric oxide (NO) is a gas with a short half-life, which is able to affect the body in a variety of pathological processes. Within the central nervous system, NO serves a dual function of neuronal protection and neurotoxicity. The present study indicated that the levels of iNOS increased significantly in sevoflurane-induced rats, suggesting the production of NO may be associated with POCD (29). In addition, it has previously been indicated that apoptosis occurring following POCD is associated with increased production of $\mathrm{NO}$ (30). A previous study demonstrated that using a selective iNOS inhibitor resulted in the reduction in the expression of caspase-3 (31). Therefore, the expression of iNOS may induce cell apoptosis, which is important in POCD (32). Notably, the current study observed that treatment with tetrandrine inhibited the increase in the levels of iNOS in the sevoflurane-treated aged rats. Previous studies have suggested that tetrandrine inhibits the activation of mesangial cells through a reduction in the expression of iNOS (33). Wang et al (34) reported that tetrandrine treatment reduced the production of iNOS and COX-2 expression in vitro and in vivo.

Bcl-2, Bax and caspase 3 are important apoptosis-related proteins in the hippocampus, which are associated with POCD. In the Bcl-2 family of proteins, Bcl-2 is an important 
anti-apoptotic protein whilst Bax promotes apoptosis (35). Bcl-2 is predominantly localized to the nuclear membrane, endoplasmic reticulum and the mitochondrial membrane. Under normal physiological conditions, Bax is located in the cytoplasm, however, following apoptotic stimuli it undergoes conformational alterations and becomes associated with the mitochondrial membrane. Bcl-2 functions as an anti-apoptotic protein by binding to Bax and preventing this process from occurring (30). Caspase-3 is a key executioner of apoptosis and its activation serves an important role in triggering downstream apoptotic processes (36). The current study indicates that tetrandrine reduced the increase in caspase- 3 activity observed in sevoflurane-treated rats. In addition, tetrandrine-treated sevoflurane-induced rats were observed to have increased expression levels of Bcl-2 compared with untreated sevoflurane-treated rats. In support of this, Gong et al (37) indicated that tetrandrine attenuated lipopolysaccharide-induced fulminant hepatic failure through a reduction in the levels of caspase-3.

In conclusion, the current study indicates that tetrandrine is able to ameliorate sevoflurane-induced cognitive impairment via the suppression of inflammation and apoptosis. This suggests that tetrandrine may be a potential candidate treatment to ameliorate cognitive impairment and alleviate neurodegeneration in sevoflurane-treated elderly patients. Further in vivo or in vitro studies are required to investigate the effects of tetrandrine on cognitive function following sevoflurane exposure, and whether there is an effect upon the interaction between neurons and microglia following treatment with tetrandrine.

\section{References}

1. Liu J, Wang P, Zhang X, Zhang W and Gu G: Effects of different concentration and duration time of isoflurane on acute and long-term neurocognitive function of young adult C57BL/6 mouse. Int J Clin Exp Pathol 7: 5828-5836, 2014.

2. Boost KA, Leipold T, Scheiermann P, Hoegl S, Sadik CD, Hofstetter C and Zwissler B: Sevoflurane and isoflurane decrease TNF-alpha-induced gene expression in human monocytic THP-1 cells: Potential role of intracellular IkappaBalpha regulation. Int J Mol Med 23: 665-671,2009.

3. Le Freche H, Brouillette J, Fernandez-Gomez FJ, Patin P, Caillierez R, Zommer N, Sergeant N, Buée-Scherrer V, Lebuffe G, Blum D and Buée L: Tau phosphorylation and sevoflurane anesthesia: An association to postoperative cognitive impairment. Anesthesiology 116: 779-787, 2012.

4. Liu Y, Pan N, Ma Y, Zhang S, Guo W, Li H, Zhou J, Liu G and Gao M: Inhaled sevoflurane may promote progression of amnestic mild cognitive impairment: A prospective, randomized parallel-group study. Am J Med Sci 345: 355-360, 2013.

5. Wan Y, Xu J, Ma D, Zeng Y, Cibelli M and Maze M: Postoperative impairment of cognitive function in rats: A possible role for cytokine-mediated inflammation in the hippocampus. Anesthesiology 106: 436-443, 2007.

6. Chen W, Liu B, Zhang F, Xue P, Cui R and Lei W: The effects of dexmedetomidine on post-operative cognitive dysfunction and inflammatory factors in senile patients. Int J Clin Exp Med 8: 4601-4605, 2015.

7. Su X, Feng X, Terrando N, Yan Y, Chawla A, Koch LG, Britton SL, Matthay MA and Maze M: Dysfunction of inflammation-resolving pathways is associated with exaggerated postoperative cognitive decline in a rat model of the metabolic syndrome. Mol Med 18: 1481-1490, 2012.

8. Fang DC and Jiang MX: Studies on tetrandrine calcium antagonistic action. Chin Med J (Engl) 99: 638-644, 1986.

9. Shi C, Ahmad Khan S, Wang K and Schneider M: Improved delivery of the natural anticancer drug tetrandrine. Int J Pharm 479: 41-51, 2015.
10. Wang Y,He H, Li D, Zhu W, Duan K, Le Y, Liao Y and Ou Y: The role of the TLR4 signaling pathway in cognitive deficits following surgery in aged rats. Mol Med Rep 7: 1137-1142, 2013.

11. Leijten FS, Alpherts WC, Van Huffelen AC, Vermeulen J and Van Rijen PC: The effects on cognitive performance of tailored resection in surgery for nonlesional mesiotemporal lobe epilepsy. Epilepsia 46: 431-439, 2005.

12. Jin Y, Zhao X, Li H, Wang Z and Wang D: Effects of sevoflurane and propofol on the inflammatory response and pulmonary function of perioperative patients with one-lung ventilation. Exp Ther Med 6: 781-785, 2013

13. Cremer J, Stoppe C, Fahlenkamp AV, Schälte G, Rex S, Rossaint R and Coburn M: Early cognitive function, recovery and well-being after sevoflurane and xenon anaesthesia in the elderly: A double-blinded randomized controlled trial. Med Gas Res 1: 9, 2011.

14. He FQ, Qiu BY, Li TK, Xie Q, Cui J, Huang XL and Gan HT: Tetrandrine suppresses amyloid- $\beta$-induced inflammatory cytokines by inhibiting NF- $\mathrm{BB}$ pathway in murine BV2 microglial cells. Int Immunopharmacol 11: 1220-1225, 2011

15. Park PH, Nan JX, Park EJ, Kang HC, Kim JY, Ko G and Sohn DH: Effect of tetrandrine on experimental hepatic fibrosis induced by bile duct ligation and scission in rats. Pharmacol Toxicol 87: 261-268, 2000.

16. Zhang J, Yu B, Zhang XQ, Sheng ZF, Li SJ, Wang ZJ, Cui XY, Cui SY and Zhang YH: Tetrandrine, an antihypertensive alkaloid, improves the sleep state of spontaneously hypertensive rats (SHRs). J Ethnopharmacol 151: 729-732, 2014.

17. Bickmeyer U, Weinsberg F, Müller E and Wiegand H: Blockade of voltage-operated calcium channels, increase in spontaneous catecholamine release and elevation of intracellular calcium levels in bovine chromaffin cells by the plant alkaloid tetrandrine. Naunyn Schmiedebergs Arch Pharmacol 357: 441-445, 1998.

18. Chen L, Chen L, Lv Y, Cui Z, Bei G, Qin G, Zhou J and Ge T: Tetrandrine ameliorates cognitive impairment via inhibiting astrocyte-derived S100B activation in a rat model of chronic cerebral hypoperfusion. Neurol Res 35: 614-621, 2013.

19. He FQ, Qiu BY, Zhang XH, Li TK, Xie Q, Cui DJ, Huang XL and Gan HT: Tetrandrine attenuates spatial memory impairment and hippocampal neuroinflammation via inhibiting NF- $\mathrm{KB}$ activation in a rat model of Alzheimer's disease induced by amyloid- $\beta(1-42)$. Brain Res 1384: 89-96, 2011.

20. Kamer AR, Galoyan SM, Haile M, Kline R, Boutajangout A, Li YS and Bekker A: Meloxicam improves object recognition memory and modulates glial activation after splenectomy in mice. Eur J Anaesthesiol 29: 332-337, 2012.

21. Li YC, Xi CH, An YF, Donh WH and Zhou M: Perioperative inflammatory response and protein S-100 $\beta$ concentrations - relationship with post-operative cognitive dysfunction in elderly patients. Acta Anesthesiol Scand 56: 595-600, 2012.

22. Cheng Q, Wang J, Wu A, Zhang R, Li L and Yue Y: Can urinary excretion rate of 8-isoprostrane and malonaldehyde predict postoperative cognitive dysfunction in aging? Neurol Sci 34: 1665-1669, 2013

23. Wu SJ and $\mathrm{Ng} \mathrm{LT}$ : Tetrandrine inhibits proinflammatory cytokines, iNOS and COX-2 expression in human monocytic cells. Biol Pharm Bull 30: 59-62, 2007.

24. Kang OH, An HJ, Kim SB, Mun SH, Seo YS, Joung DK, Choi JG, Shin DW and Kwon DY: Tetrandrine suppresses pro-inflammatory mediators in PMA plus A23187-induced HMC-1 cells. Int J Mol Med 33: 1335-1340, 2014.

25. Hu Z, Ou Y, Duan K and Jiang X: Inflammation: A bridge between postoperative cognitive dysfunction and Alzheimer's disease. Med Hypotheses 74: 722-724, 2010.

26. Windham BG, Simpson BN, Lirette S, Bridges J, Bielak L, Peyser PA, Kullo I, Turner S, Griswold ME and Mosley TH: Associations between inflammation and cognitive function in African Americans and European Americans. J Am Geriatr Soc 62: 2303-2310, 2014.

27. Lin ST, Wang Y, Xue Y, Feng DC, Xu Y and Xu LY: Tetrandrine suppresses LPS-induced astrocyte activation via modulating IKKs-IkappaBalpha-NF-kappaB signaling pathway. Mol Cell Biochem 315: 41-49, 2008.

28. Zhang DK, Cheng LN, Huang XL, Shi W, Xiang JY and Gan HT: Tetrandrine ameliorates dextran-sulfate-sodium-induced colitis in mice through inhibition of nuclear factor-kappaB activation. Int J Colorectal Dis 24: 5-12, 2009.

29. Tang N, Ou C, Liu Y, Zuo Y and Bai Y: Effect of inhalational anaesthetic on postoperative cognitive dysfunction following radical rectal resection in elderly patients with mild cognitive impairment. J Int Med Res 42: 1252-1261, 2014. 
30. Qian XL, Zhang W, Liu MZ, Zhou YB, Zhang JM, Han L, Peng YM, Jiang JH and Wang QD: Dexmedetomidine improves early postoperative cognitive dysfunction in aged mice. Eur J Pharmacol 746: 206-212, 2015.

31. Kiang JG, Aggravante NG, Smith JT and Bowman PD: 17-DMAG diminishes hemorrhage-induced small intestine injury by elevating $\mathrm{Bcl}-2$ protein and inhibiting iNOS pathway, TNF- $\alpha$ increase, and caspase-3 activation. Cell Biosci 1l: 21 , 2011.

32. Jia M, Liu WX, Sun HL, Chang YQ, Yang JJ, Ji MH, Yang JJ and Feng CZ: Suberoylanilide hydroxamic acid, a histone deacetylase inhibitor, attenuates postoperative cognitive dysfunction in aging mice. Front Mol Neurosci 8: 52, 2015.

33. Wu CJ, Wang YH, Lin CJ, Chen $\mathrm{HH}$ and Chen YJ: Tetrandrine down-regulates ERK/NF- $\mathrm{BB}$ signaling and inhibits activation of mesangial cells. Toxicol In Vitro 25: 1834-1840, 2011.
34. Wang QS, Cui YL, Gao LN, Guo Y, Li RX and Zhang XZ: Reduction of the pro-inflammatory response by tetrandrine-loading poly(L-lactic acid) films in vitro and in vivo. J Biomed Mater Res A 102: 4098-4107, 2014.

35. Li SY, Xia LX, Zhao YL, Yang L, Chen YL, Wang JT and Luo AL: Minocycline mitigates isoflurane-induced cognitive impairment in aged rats. Brain Res 1496: 84-93, 2013.

36. Chen R, Liu S, Piao F, Wang Z, Qi Y, Li S, Zhang D and Shen J: 2,5-hexanedione induced apoptosis in mesenchymal stem cells from rat bone marrow via mitochondria-dependent caspase-3 pathway. Ind Health 53: 222-235, 2015.

37. Gong X, Luo FL, Zhang L, Li HZ, Wu MJ, Li XH, Wang B, $\mathrm{Hu}$ N, Wang CD, Yang JQ and Wan JY: Tetrandrine attenuates lipopolysaccharide-induced fulminant hepatic failure in D-galactosamine-sensitized mice. Int Immunopharmacol 10: 357-363, 2010 